\title{
On the Social Cost of Distributed Selfish Content Replication
}

\author{
Gerasimos G. Pollatos $^{1, \star}$, Orestis A. Telelis ${ }^{2, \star \star}$, and Vassilis Zissimopoulos ${ }^{1}$ \\ ${ }^{1}$ Department of Informatics and Telecommunications, University of Athens, Greece \\ \{gpol,vassilis\}@di.uoa.gr \\ ${ }^{2}$ Department of Computer Science, University of Aarhus, Denmark \\ telelis@daimi.au.dk
}

\begin{abstract}
We study distributed content replication networks formed voluntarily by selfish autonomous users, seeking access to information objects that originate from distant servers. Each user caters to minimization of its individual access cost by replicating locally (up to constrained storage capacity) a subset of objects, and accessing the rest from the nearest possible location. We show existence of stable networks by proving existence of pure strategy Nash equilibria for a game-theoretic formulation of this situation. Social (overall) cost of stable networks is measured by the average or by the maximum access cost experienced by any user. We study socially most and least expensive stable networks by means of tight bounds on the ratios of the Price of Anarchy and Stability respectively. Although in the worst case the ratios may coincide, we identify cases where they differ significantly. We comment on simulations exhibiting occurence of cost-efficient stable networks on average.
\end{abstract}

\section{Introduction}

Distributed network storage constitutes a frequently exploited modern resource for the improvement of services offered to internet users. A network node can replicate locally content (files or services) that is accessed frequently by local users, so as to lessen bandwidth consumption incurred by frequent remote access. Non-locally replicated objects can be retrieved from the nearest location where they can be found replicated (neighbors/origin servers). Web caching, content distribution networks and, more recently, applications over P2P networks serve this scheme. Query protocols [1, succint summaries [2], and distributed hash tables [3] can implement search of object replicas at remote nodes.

Such systems are frequently modeled as Distributed Replication Groups [4]: a distant server distributes content represented as a set of information objects to a network of nodes sharing proximity, each having constrained local storage

\footnotetext{
* Author supported by the 03ED694 research project (PENED), co-financed by E.U.European Social Fund (75\%) and the Greek Ministry of Development-GSRT (25\%).

** Center for Algorithmic Game Theory, funded by the Carlsberg Foundation, Denmark.
} 
capacity. Each node has an interest to a subset of information objects in the form of a non-negative demand weight per object. Access cost incurred per non-locally replicated object is defined as the distance from which the object is retrieved times the demand weight. The related Object (or Data) Placement problem requires placement of object replicas in nodes, subject to capacity constraints, so that total access cost summed over all objects and nodes is minimized 45].

We consider networks formed voluntarily by autonomous users for shared exploitation of distributed resources (storage capacity), such as file-sharing networks. We develop a game-theoretic analysis of distributed replication networks, in the light of which, users choose autonomously a replication strategy (a subset of objects to replicate locally), catering to minimization of their individual access cost. Through our study of the Distributed Selfish Replication game we address systematically the questions: "Does the performance of the network ever stabilize?" and "What is the overall performance of a stable network, in absence of a central optimizing authority?" Game theory predicts that users will eventually end up (converge) in a Nash equilibrium [6], i.e. a set of (randomized in general) replication strategies, under which no user can decrease his/her individual (in general, expectation of) access cost by unilaterally changing his/her strategy [7.

In view of equilibria as a notion of network stabilization, we are interested in deterministic strategies (pure equilibria), because randomized strategies do not naturally fit behavior of a rational user [78, as he/she may observe inefficiency during network's lifetime and change the replication strategy. Two natural ways of measuring the social (overall) network cost include the average cost experienced by network users (SUM of individual costs), and the maximum cost over all users (MAX). The coordination ratio of such systems, widely known as the Price of Anarchy (PoA) was introduced in the seminal work of [9] as the worst-case ratio of the social cost of the most expensive equilibrium over the optimum centrally enforcable social cost. The PoA measures worst-case performance loss due to the absense of a centralized coordinating authority. More recently in [10] it was proposed that the worst-case ratio of the social cost of the least expensive equilibrium to the optimum social cost is also of interest, since some kind of coordination may be provided to the users, so that they reach the most efficient stable state. This ratio, initially termed the optimistic PoA [10, was established in 11] as the Price of Stability, (PoS) i.e. the performance loss due to the requirement for stability.

In this paper we prove existence of pure strategy Nash equilibria for the Distributed Selfish Replication game, (section 3) over a slightly more general network model than the one originally proposed in [4, by analyzing a converging best response dynamics algorithm. We prove a tight upper bound for the PoA and matching lower bounds for the PoS (section 4), for both the SUM and MAX social costs: our PoA upper bound is valid for more general network models than the one considered. However, the simple model that we study exhibits tight worst-case lower bounds for the PoS. We identify cases where the PoS can be significantly smaller than PoA, and comment on simulations that exhibit occurence of cost-efficient equilibria on average (section 5). Our results generalize 
and complement the work of [12], and answer a related open question posed in [8] with respect to considering constrained storage capacity in replication games.

Related Work. The study of voluntarily formed data replication networks by autonomous users with individual incentives was initiated in [8], where each replicated object could be payed for a fixed amount (as opposed to capacity constraints). It was shown that both PoA and PoS can be unbounded in general with the exception of special topologies. The authors considered only $0 / 1$ demand weights in their analysis. They posed consideration of capacitated nodes along with demand weights as an open question. Let us note here that the notion of capacity should not be perceived under the narrow view of hard disk storage (which is quite affordable nowadays), but rather as a more general notion of budget, to be spent for maintenance and reliability purposes (particularly when replication concerns services). Existence of equilibria for the simple capacitated model of [4] was shown in [12], and for demand weights that constitute a distribution over the set of objects requested by each node. This work however does not concern social efficiency, but rather maximization of the gain of individuals when participating in the network as opposed to staying isolated. Our work is strongly related to the recent studies on network formation games initiated by the seminal work of 13 , in an effort of understanding the efficiencies and defficiencies induced to a network created by autonomous users, that are motivated by colliding incentives. A wealth of recent results on such network creation models [11014 have grown this direction of research into an exciting field [15] (chapter 19), that has provided useful insights with respect to the manipulation of the users' incentives towards creation of socially efficient networks.

\section{Preliminaries}

We consider a network involving $k$ servers and a set $N,|N|=n$ of client-nodes (simply referred to as nodes), each node having at its disposal integral local storage capacity of size $_{i}$. A universe $U$ of up-to-date unit-sized information objects originates from the $k$ servers and each node $i$ requests access to each object $o \in U$ by means of a demand weight $w_{i o} \geq 0$. The request set of $i$ is $R_{i}=\left\{o \in U \mid w_{i o}>0\right\}$. Node $i$ may choose to replicate locally any at most size $i^{-}$ cardinality subset of $R_{i}$, and access every non-replicated object $o$ from some node (or server) $j \neq i$ at cost $d_{i j} w_{i o}$, where $d_{i j}$ is the cost (distance) of accessing $j$ per unit of demand.

If $P_{i}$ is the subset of objects replicated by $i$, a placement over the network is denoted by $X=\left\{P_{1}, \ldots, P_{n}\right\}$. Given a placement $X$, we use $d_{i}(o)$ to denote the distance from node $i$ of the closest node (including origin servers: they can be considered as nodes with fixed placements) replicating object $o$. Formally: $d_{i}(o)=\min _{j \neq i: o \in P_{j}} d_{i j}$. The individual access cost of $i$ under a placement $X$, is defined as:

$$
c_{i}(X)=\sum_{o \in R_{i} \backslash P_{i}} w_{i o} d_{i}(o)
$$


Our study involves two versions of social (overall) cost: SUM and MAX, defined as $\sum_{i} c_{i}(X)$ and $\max _{i} c_{i}(X)$ respectively. We study the strategic Distributed Selfish Replication (DSR, and DSR(0/1) when demand weights are 0 or 1 ) game defined by the triple $\left\langle N,\left\{P_{i}\right\},\left\{c_{i}\right\}\right\rangle$ in which every node $i$ is a player with strategy space $\left\{P_{i}\right\}$ consisting of all size $e$-cardinality subsets of objects that $i$ may replicate, and utility expressed by the access cost $c_{i}$, that $i$ wishes to minimize. A placement $X$ is then a strategy profile for the DSR game. In what follows we use the terms node and player interchangeably. Let $X_{-i}=\left\{P_{1}, \ldots, P_{i-1}, P_{i+1}, \ldots, P_{n}\right\}$ refer to the strategy profile of all players but $i$.

For the DSR game, it is easy to see that given a strategy profile $X_{-i}$, player $i$ can determine optimally in polynomial time its best response $P_{i}$ to the other players' strategies, simply by solving a special 0/1 Knapsack problem, that amounts to replicating size $e_{i}$ objects with the greatest value $w_{i o} d_{i}(o)$.

Network Model. We consider a slightly more general network model than the one introduced by Leff,Wolf, and $\mathrm{Yu}$ in [4] and studied in 12, involving $k$ origin servers, instead of 1 . The distance of every node $i$ from server $l$ is $d_{l}$ for $l=0 \ldots k-1$, while two nodes $i$ and $j$ are at distance $d_{i j}=d_{k}$. We assume that distances form an ultra-metric such that $d_{k}<d_{k-1}<\cdots<d_{0}$. We refer to this network model as $\mathbf{L W Y}(k)$. The minimum and maximum distances appearing in the network are also referred to with $d_{\min }=d_{k}$ and $d_{\max }=d_{0}$.

\section{Existence of Pure Strategy Nash Equilibria}

We introduce a simple polynomial-time algorithm, that finds a feasible placement constituting a pure strategy Nash equilibrium for the DSR game on a LWY $(k)$ networks. The algorithm constitutes a converging iterative best response dynamics that iterates for $l=0 \ldots k$ over the different distance values (in non-increasing order) appearing in $\mathbf{L W Y}(k)$ : for each distance value $d_{l}$, all distances at most equal to $d_{l}$ are redefined to exactly $d_{l}$ and a best response placement is computed for each node $i=1 \ldots n$ in turn. It is important to note that during computation of best response of a node $i$ a tie for the values of two objects $w_{i o} d_{i}(o)$ and $w_{i o^{\prime}} d_{i}\left(o^{\prime}\right)$ is resolved on the basis of index (for otherwise we cannot guarantee convergence to equilibrium). The order by which nodes compute their best response is fixed in all iterations. We refer to this algorithm (algorithm 1) as DSR-EQ. We show that DSR-EQ finds an equilibrium placement. In our analysis we use the iteration index $l$ as superscript to the various quantities involved.

Proposition 1. Consider the time right after computation of placements $P_{i}^{l-1}$ and $P_{i}^{l}$ for a fixed node $i$ in iterations $l-1$ and $l$ of algorithm DSR-EQ. Then for every pair of objects $o, o^{\prime}$ with $o \in P_{i}^{l-1} \backslash P_{i}^{l}$ and $o^{\prime} \in P_{i}^{l} \backslash P_{i}^{l-1}$ it is $d_{i}^{l}(o)=d_{l}$ and $d_{i}^{l}\left(o^{\prime}\right)>d_{l}$.

The proof is by induction on indices $\langle i, l\rangle$ through the following claims:

Claim. (Basic Step) Proposition 1 holds for $l=1$ (second iteration) and $i=1$. 


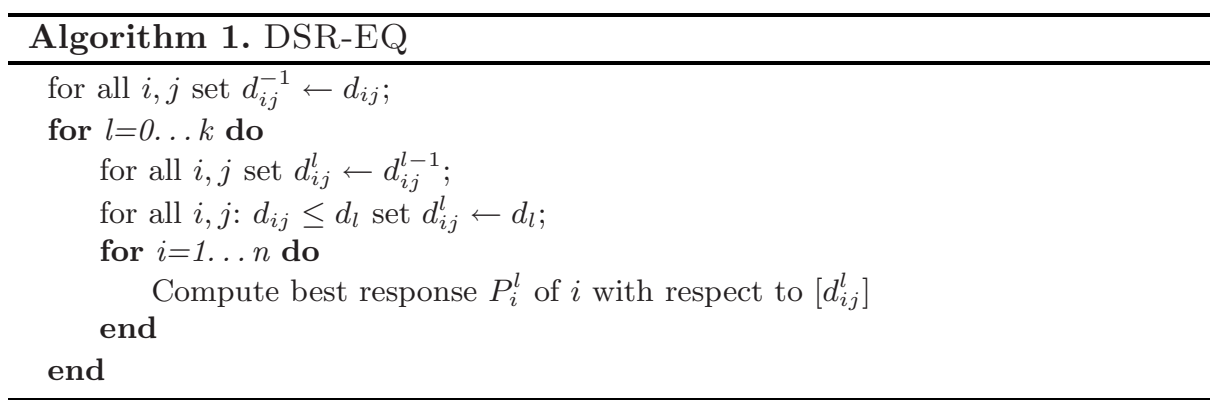

Proof. Right after computation of $P_{1}^{0}$ and $P_{1}^{1}$ the following hold with respect to objects $O$ and $o^{\prime}$ :

$$
d_{1}^{0}\left(o^{\prime}\right) w_{1 o^{\prime}} \leq d_{1}^{0}(o) w_{1 o}, \quad \text { and } \quad d_{1}^{1}\left(o^{\prime}\right) w_{1 o^{\prime}} \geq d_{1}^{1}(o) w_{1 o}
$$

Equality in both cases cannot hold, because of the index rule for breaking ties. From the first inequality it must be that $w_{1 o^{\prime}} \leq w_{1 o}$ because $d_{1}^{0}\left(o^{\prime}\right)=d_{1}^{0}(o)=d_{0}$. Furthermore, in iteration $l=1$, by redefinition of distances less than $d_{0}$ in the original input to $d_{1}$, it must be either that $d_{1}^{1}\left(o^{\prime}\right)=d_{1}^{1}(o)=d_{0}$ (both objects are found only at server distance $\left.d_{0}\right)$, or that $d_{1}^{1}\left(o^{\prime}\right)=d_{1}^{1}(o)=d_{1}$ (both objects are replicated somewhere at distance $\left.d_{1}\right)$, or that $d_{1}^{1}\left(o^{\prime}\right) \neq d_{1}^{1}(o)$. The first two imply $w_{1 o^{\prime}} \geq w_{1 o}$ which contradicts $w_{1 o^{\prime}} \leq w_{1 o}$, even in the case of equality (because of the tie-breaking index rule). For the third case, if $d_{1}^{1}\left(o^{\prime}\right)=d_{1}$ and $d_{1}^{1}(o)=d_{0}$, we obtain that $d_{1}^{1}\left(o^{\prime}\right) w_{1 o^{\prime}}<d_{1}^{1}(o) w_{1 o}$, a contradiction to the second relation of (2). Thus it must be that $d_{1}^{1}\left(o^{\prime}\right)=d_{0}>d_{1}$ and $d_{1}^{1}(o)=d_{1}$.

Claim. (Inductive Step) Proposition 1 holds for every iteration $l$.

Proof. Assume that the statement is true for all nodes $\{i+1, \ldots, n, 1, \ldots, i-1\}$ computing their best response right after computation of $P_{i}^{l-1}$ and right before computation of $P_{i}^{l}$. For $o \in P_{i}^{l-1} \backslash P_{i}^{l}$ and $o^{\prime} \in P_{i}^{l} \backslash P_{i}^{l-1}$ we can write:

$$
d_{i}^{l-1}\left(o^{\prime}\right) w_{i o^{\prime}} \leq d_{i}^{l-1}(o) w_{i o}, \quad \text { and } \quad d_{i}^{l}\left(o^{\prime}\right) w_{i o^{\prime}} \geq d_{i}^{l}(o) w_{i o}
$$

Equality in both cases cannot occur as before. Assume that $d_{i}^{l}(o)>d_{l}$. Then $d_{i}^{l}(o) \geq d_{l-1}$ and, if $o$ was replicated at some node in iteration it $l-1$ (i.e. $\left.d_{i}^{l-1}(o)=d_{l-1}\right)$, it must have remained so, by hypothesis. But then it should have been $d_{i}^{l}(o)=d_{l}$. Thus $o$ was found only at some server in iteration $l-1$. Then $d_{i}^{l}(o)=d_{i}^{l-1}(o)$. For $o^{\prime}$ however, we have that $d_{i}^{l}\left(o^{\prime}\right) \leq d_{i}^{l-1}\left(o^{\prime}\right)$, because of the assumed behavior of the other nodes computing best response after $P_{i}^{l-1}$ and before $P_{i}^{l}$ : either $o^{\prime}$ reduces its distance from $i$ due to redefinition of distances to $d_{l}$ (in case some node has it replicated) or not. Then it is:

$$
d_{i}^{l}\left(o^{\prime}\right) w_{i o^{\prime}} \leq d_{i}^{l-1}\left(o^{\prime}\right) w_{i o^{\prime}} \leq d_{i}^{l-1}(o) w_{i o}=d_{i}^{l}(o)
$$

which is a contradiction to (3). Thus it must be $d_{i}^{l}(o)=d_{l}$. 
Now assume that $d_{i}^{l}\left(o^{\prime}\right)=d_{l}$. Since $d_{i}^{l}(o)=d_{l}$ also, we have that $w_{i o^{\prime}} \geq w_{i o}$. Furthermore, in iteration $l-1$ it must have been $d_{i}^{l-1}\left(o^{\prime}\right) \geq d_{l-1}$ and $d_{i}^{l-1}(o)=$ $d_{l-1}$. Then we obtain $d_{i}^{l-1}\left(o^{\prime}\right) w_{i o^{\prime}} \geq d_{i}^{l}\left(o^{\prime}\right) w_{i o^{\prime}}=d_{l} w_{i o^{\prime}}>d_{l} w_{i o}=d_{i}^{l}(o) w_{i o}=$ $d_{i}^{l-1}(o) w_{i o}$, which is a contradiction to (3). Thus it must be $d_{i}^{l}\left(o^{\prime}\right)>d_{l}$.

Using proposition 1 it is possible to show that:

Lemma 1. In the end of iteration $l$ of algorithm $1,\left\{P_{i}^{l} \mid i=1 \ldots n\right\}$ is a pure strategy Nash equilibrim with respect to the current distance matrix $\left[d_{i j}^{l}\right]$.

Proof. By proposition 1 we deduce that, in each iteration, a node's best response is not invalidated by other nodes' best responses, as follows. In the $l$-th iteration, for every node $j \neq i$ and an object $o \in P_{j}^{l-1} \backslash P_{j}^{l}$ the object remains at distance $d_{l}$ from $i$ (which was its distance when $i$ was computing $P_{i}^{l}$ ). For an object $o^{\prime} \in P_{j}^{l} \backslash P_{j}^{l-1}, o^{\prime}$ was at distance $>d_{l}$ and, therefore, not replicated in $i$ either. Replication in $j$ only decreases the value of $o^{\prime}$ for $i$ (because its distance is reduced).

Theorem 1. Pure strategy Nash equilibria exist for the DSR game on $\boldsymbol{L W} \boldsymbol{Y}(k)$ networks.

Proof. By lemma 1 the placement produced in the last iteration of algorithm 1 is a pure strategy Nash equilibrium with respect to a distance matrix identical to the input one.

\section{Worst-Case Equilibria}

We study efficiency of pure equilibria for the DSR game, by studying the worstcase ratios of the PoA and PoS. We assume that all players (nodes) have a marginal interest for joining the network, expressed by $\left|R_{i}\right| \geq s i z e_{i}$. If this does not hold, then equilibria may be unboundedly inefficient, because of unused storage space in certain nodes. In summary we show that the PoA can be at most $\frac{d_{\max }}{d_{\min }}$ for both the SUM and the MAX social cost functions, and the PoS can be at least as much in the worst-case. We compare a worst-case socially most expensive equilibrium placement $X$ towards the socially optimum placement $X^{*}$, and use $d_{i}(\cdot)$ and $d_{i}^{*}(\cdot)$ for distances of objects in each placement. The analysis is performed for the individual cost of a single fixed node $i$, and therefore yields results with respect to the SUM and the MAX social cost functions.

Lemma 2. If $X$ and $X^{\star}$ are equilibrium and optimum placements respectively, for each node $i$ we have:

$$
\begin{aligned}
c_{i}(X) & =\sum_{o \in R_{i} \backslash\left(P_{i} \cup P_{i}^{\star}\right)} w_{i o} d_{i}(o)+\sum_{o \in P_{i}^{\star} \backslash P_{i}} w_{i o} d_{i}(o) \\
c_{i}\left(X^{\star}\right) & =\sum_{o \in R_{i} \backslash\left(P_{i}^{\star} \cup P_{i}\right)} w_{i o} d_{i}^{\star}(o)+\sum_{o \in P_{i} \backslash P_{i}^{\star}} w_{i o} d_{i}^{\star}(o)
\end{aligned}
$$


Proof. Taking expression (1) for $c_{i}$ at $X$ and $X^{\star}$ and noticing that:

$$
\begin{aligned}
& R_{i} \backslash P_{i}=\left(R_{i} \backslash\left(P_{i} \cup P_{i}^{\star}\right)\right) \cup\left(P_{i}^{\star} \backslash P_{i}\right) \text { and }\left(R_{i} \backslash\left(P_{i} \cup P_{i}^{\star}\right)\right) \cap\left(P_{i}^{\star} \backslash P_{i}\right)=\emptyset \\
& R_{i} \backslash P_{i}^{\star}=\left(R_{i} \backslash\left(P_{i}^{\star} \cup P_{i}\right)\right) \cup\left(P_{i} \backslash P_{i}^{\star}\right) \text { and }\left(R_{i} \backslash\left(P_{i}^{\star} \cup P_{i}\right)\right) \cap\left(P_{i} \backslash P_{i}^{\star}\right)=\emptyset
\end{aligned}
$$

yields the result.

Lemma 3. If $X$ and $X^{\star}$ are equilibrium and optimum placements respectively, for every player $i$ we have that $\left|P_{i} \backslash P_{i}^{\star}\right|=\left|P_{i}^{\star} \backslash P_{i}\right|$ and for every pair of objects $o \in P_{i} \backslash P_{i}^{\star}, o^{\prime} \in P_{i}^{\star} \backslash P_{i}$ it is $w_{i o} d_{i}(o) \geq w_{i o^{\prime}} d_{i}\left(o^{\prime}\right)$.

Proof. Since for every player $i$ size $e_{i}<\left|R_{i}\right|$, we obtain $\left|P_{i} \backslash P_{i}^{\star}\right|=\left|P_{i}^{\star} \backslash P_{i}\right|$. For every pair of objects $o \in P_{i} \backslash P_{i}^{\star}, o^{\prime} \in P_{i}^{\star} \backslash P_{i}$ it must be $w_{i o} d_{i}(o)>w_{i o^{\prime}} d_{i}\left(o^{\prime}\right)$, for otherwise, player $i$ would have an incentive to unilaterally deviate in placement $X$, by substituting $o$ for $o^{\prime}$, thus decreasing its individual access cost.

Theorem 2. The price of anarchy for the DSR game is upper bounded by $\frac{d_{\max }}{d_{\min }}$, with respect to either of the SUM or the MAX social cost functions.

Proof. Starting from expression (5) and using lemma 3 we have:

$$
\begin{aligned}
c_{i}\left(X^{\star}\right) & =\sum_{o \in R_{i} \backslash\left(P_{i}^{\star} \cup P_{i}\right)} w_{i o} d_{i}^{\star}(o)+\sum_{o \in P_{i} \backslash P_{i}^{\star}} w_{i o} d_{i}^{\star}(o) \\
& \geq \sum_{o \in R_{i} \backslash\left(P_{i}^{\star} \cup P_{i}\right)} w_{i o} d_{i}^{\star}(o) \frac{d_{i}(o)}{d_{i}(o)}+\sum_{o^{\prime} \in P_{i}^{\star} \backslash P_{i}} w_{i o^{\prime}} \frac{d_{i}\left(o^{\prime}\right)}{d_{i}(o)} d_{i}^{\star}(o) \\
& \geq \sum_{o \in R_{i} \backslash\left(P_{i}^{\star} \cup P_{i}\right)} w_{i o} d_{i}(o) \frac{d_{\text {min }}}{d_{\text {max }}}+\sum_{o^{\prime} \in P_{i}^{\star} \backslash P_{i}} w_{i o^{\prime}} d_{i}\left(o^{\prime}\right) \frac{d_{\text {min }}}{d_{\text {max }}}
\end{aligned}
$$

By expression (4) $c_{i}\left(X^{\star}\right) \geq \frac{d_{\min }}{d_{\max }} c_{i}(X)$. Summing over all $i$ yields the result for the SUM. For the MAX notice that for some $j$ : $\max _{i} c_{i}(X)=c_{j}(X) \leq \frac{d_{\max }}{d_{\min }} c_{j}\left(X^{*}\right) \leq$ $\frac{d_{\max }}{d_{\min }} \max _{i} c_{i}\left(X^{*}\right)$.

Note that we did not make use of any particular network topology. The bound is valid on any network topology, provided an equilibrium exists. Interestingly, matching lower bounds for the PoS can be shown on a LWY(1) network:

Proposition 2. The Price of Stability for the DSR game has a lower bound arbitrarily close to $d_{\max } / d_{\min }$ in the worst-case, with respect to SUM and MAX social cost functions, even for 1 server and 0/1 demand weights.

Proof. Take $d_{\text {min }}$ inter-node distance and $d_{\max }$ the distance of the single server.

For SUM: we show that for every fixed integer $I$ the PoS is lower bounded by $\frac{I}{I+1} \frac{d_{\max }}{d_{\min }}$ asymptotically with $n$. Take size $e_{i}=1$ and set: $R_{i}=\{a\}$ for $i=$ $1 \ldots n-I$ and $R_{i}=\left\{b_{j} \mid j=1 \ldots n-1\right\}$, for $i=n-I+1 \ldots n$. In every equilibrium 
of this instance the $I$ last nodes replicate collectively at most $I$ objects from their (common) request set, while the rest $n-I$ replicate object $a$. The social cost is $I(I-1) d_{\min }+I(n-1-I) d_{\max }$. In the socially optimum placement we let nodes $i=1 \ldots n-I-1$ replicate $n-I-1$ objects that are not replicated in the $I$ last nodes. But, we save a node for replicating $a$. The social cost becomes $I(n-2) d_{\min }+(n-1-I) d_{\min }=[(I+1) n-3 I-1] d_{\min }$. Since $I$ is fixed, the PoS becomes $\frac{I}{I+1} \frac{d_{\max }}{d_{\min }}$ as $n$ grows.

For MAX: all nodes have size $_{i}=1$ and let $R_{1}=\{a, b\}$, whereas for $i>1$ set $R_{i}=\{c\}$. Take $n \geq 3$ nodes. In equilibrium node 1 pays $d_{\max }$ for either $a$ or $b$. In the social optimum some $i>1$ replicates one of $a, b$ that is not replicated by node 1 , and pays $d_{\min }$ for $c$, while 1 also pays $d_{\min }$.

\section{Less Expensive Equilibria}

Proposition 2 states that the least expensive and the most expensive equilibria can be of the same cost in the worst case. In this section we investigate existence of less expensive equilibria than the ones indicated by the worst-case PoA and PoS. In particular, we elaborate on conditions under which the PoS can be at most $\frac{d_{\max }}{2 d_{\min }}$ for the $\operatorname{DSR}(0 / 1)$ game, and present some indicative experimental results on random instances of the DSR game, showing that social efficiency of equilibria does not degrade much as the ratio $d_{\max } / d_{\min }$ grows. For the rest of this section's theoretical analysis we turn our attention to the $\operatorname{DSR}(0 / 1)$ game and restrict our discussion to the SUM social cost function.

In proving proposition 2 we used "over-demanding" nodes, each requesting $O(n)$ times more objects, than the storage capacity they offer to the group. In what follows we will assume modestly demanding nodes. We define such nodes formally through the demand ratio $q=\max _{i} \frac{\left|R_{i}\right|-\text { size }_{i}}{\text { size }_{i}}$ as being $q=O(1)$. We will also use the assumption that the absolute difference of any pair of distinct distance values in the network is at least $d_{\min }$. This is e.g. the case when the network is modeled as a graph with inter-node distance being one hop, while the nearest server being at least 2 hops away.

We will compare the least expensive equilibrium $X=\left\{P_{i}\right\}$ against the socially optimum placement $X^{*}=\left\{P_{i}^{*}\right\}$, so as to obtain the maximum possible difference $c(X)-c\left(X^{*}\right)$. We consider objects $a^{*} \in P_{i}^{*} \backslash P_{i}$, that we call insertions and study an imaginary procedure that transforms $X$ into $X^{*}$, by performing such insertions. An insertion is termed significant if it contributes to $c(X)-c\left(X^{*}\right)$.

Lemma 4. If $r$ significant insertions occur in comparing a least expensive equilibrium $X$ to a socially optimum placement $X^{*}$, and at most $n_{0}$ nodes benefit by any insertion, then: $c(X)-c\left(X^{*}\right) \leq r n_{0}\left(d_{\max }-d_{\min }\right)-r d_{\min }$.

Proof. Take $a^{*} \in P_{i}^{*} \backslash P_{i}$ for some node $i$. There is an object $a \in P_{i} \backslash P_{i}^{*}$ evicted from $i$ due to insertion of $a^{*}$. We use $d_{i}(\cdot)$ to denote the current distance of an object from $i$. Our target is to show that every significant insertion contributes a social cost increase at least $d_{\text {min }}$ while potentially offering at most $\left(d_{\max }-d_{\min }\right)$ cost decrease to some nodes. At first suppose that $w_{i a^{*}}=0: i$ suffers an access 
cost increase of at least $d_{\min }$, because $a$ has to be retrieved remotely at minimum distance $d_{\text {min }}$. Every node $j \neq i$ with $w_{j a^{*}}=1$ benefits at most $\left(d_{\max }-d_{\min }\right)$ though. This is a significant insertion. Now consider the case $w_{i a^{*}}=1$. Then $d_{i}(a) \geq d_{i}\left(a^{*}\right)$, because $X$ is equilibrium. We examine the following cases:

1. $d_{i}(a)=d_{i}\left(a^{*}\right)=d_{\min }$ : then $i$ does not benefit or loose, and the insertion is indifferent to every other node $j \neq i$ with $w_{j a^{*}}=1$ (they also do not benefit or loose, because $a^{*}$ is already replicated somewhere in the network). Such an insertion is not significant.

2. $d_{i}(a)>d_{i}\left(a^{*}\right)=d_{\min }$ : the cost of $i$ increases by at least $2 d_{\min }$ (due to eviction of $a$ and $\left.d_{i}(a)-d_{i}\left(a^{*}\right) \geq d_{\min }\right)$ and decreases its cost by at most $d_{\min }$ (due to insertion of $a^{*}$ ). Such an insertion is potentially significant.

3. $d_{i}(a)=d_{i}\left(a^{*}\right)>d_{\min }: i$ does not benefit or loose, but any node $j \neq i$ with $w_{i a}=1$ and $w_{i a^{*}}=0$ looses at least $d_{i}\left(a^{*}\right)-d_{\min }>d_{\min }$ (as long as $a$ was stored in $i$ it was retrieved by $j$ from distance $\left.d_{\min }\right)$. If no such node exists, then we have a new equilibrium, potentially less expensive than $X$, a contradiction. Such an insertion can be significant.

4. $d_{i}(a)>d_{i}\left(a^{*}\right)>d_{\min }$ : eviction of $a$ from $i$ is caused at distance $d_{i}(a)$ which is at least by $d_{\text {min }}$ greater than $d_{i}\left(a^{*}\right)$. Thus $i$ looses at least $d_{\min }$ in this case. This insertion can be significant.

Notice that the cost increase effect of cases (2), (3) and (4) may be compensated by a re-insertion of the evicted object $a$ in some node $j \neq i$. By the previous arguments, such a re-insertion causes eviction of some object $a^{\prime}$ from $j$ and therefore, induces a new cost increase of at least $d_{\text {min }}$, or a new equilibrium contradiction. Such re-insertions are insignificant, as they may form chains of "movements" of objects around the network's nodes, always carrying a cost increase, but no decrease. If $n_{0}$ nodes benefit from any of the significant insertions, then assuming that all of them benefit from all the significant insertions only enlarges the difference $c(X)-c\left(X^{*}\right)$, and the result follows.

Lemma 5. The cost of the socially least expensive equilibrium placement $X$ for the $\operatorname{DSR}(0 / 1)$ game on a $\boldsymbol{L} \boldsymbol{W} \boldsymbol{Y}(k)$ network in the worst case is at least:

$$
c(X) \geq n_{0}\left(n_{0}-1\right) S d_{\min }+n_{0} r d_{\max }
$$

where $r$ is the number of significant insertions, $S$ is the minimum storage capacity, and $n_{0}$ is the number of nodes that benefit from all insertions.

Proof. For every node $j$ that benefits from an insertion $a^{*}$ it is $w_{j a^{*}}=1$, and for maximum benefit $\left(d_{\max }-d_{\min }\right)$, this object is payed $d_{\max }$ in the best equilibrium. In the worst case all $n_{0}$ nodes benefit from all $r$ significant insertions, as argued in lemma 4 (this assumption enlarges the difference $c(X)-c\left(X^{*}\right)$ ). The second term of inequality (6) is thus justified.

For the first term, we notice that since all $n_{0}$ nodes benefit from an insertion $a^{*}$, then in the least expensive equilibrium $X$ every object stored in these nodes is of interest to at least $n_{0}$ nodes of the network. Otherwise, we show that there 
may be an equilibrium of less cost: take for example an instance where every one of the $n_{0}$ nodes is either interested to replicas stored in the rest $n_{0}-1$ of them or not, and no other nodes are interested in replicas stored in these $n_{0}$ nodes. If there is a node $j$ not interested to a replica $a$ stored in some node $i$, then substitution of $a$ for $a^{*}$ in $i$ yields a less expensive equilibrium. We assume that all $n_{0}$ nodes benefiting from the $r$ insertions are also interested in objects replicated in them in equilibrium. This yields a lower bound of $n_{0}\left(n_{0}-1\right) S d_{\text {min }}$ cost.

Now we can prove the following:

Theorem 3. The Price of Stability of the $\operatorname{DSR}(0 / 1)$ game on $\boldsymbol{L} \boldsymbol{W} \boldsymbol{Y}(k)$ networks with modestly demanding nodes and distance differences at least $d_{\min }$ is upper bounded by $\frac{d_{\max }}{2 d_{\min }}$.

Proof. By lemma 4

$$
P o S \leq \frac{c(X)}{c(X)+r d_{\min }-r\left(d_{\max }-d_{\min }\right)}
$$

This bound is a monotonically decreasing function of $c(X)$. Therefore we use for $c(X)$ the lower bound given by (6) for worst-case $P o S$, and obtain:

$$
P o S \leq \frac{n_{0}\left(n_{0}-1\right) S d_{\min }+n_{0} r d_{\max }}{\left(r+r n_{0}+n_{0}\left(n_{0}-1\right) S\right) d_{\min }}
$$

By the analysis of lemma [5] the number of insertions occurring is upper bounded as $r \leq\left(q-\left(n_{0}-1\right)\right) S$. Taking the maximum value $r=\left(q-\left(n_{0}-1\right)\right) S$ increases the ratio for every value of $n_{0}$ :

$$
\begin{gathered}
P o S \leq \frac{n_{0}\left(n_{0}-1\right) S d_{\min }+n_{0}\left(q-\left(n_{0}-1\right)\right) S d_{\max }}{\left[\left(q-\left(n_{0}-1\right)\right) S+n_{0}\left(q-\left(n_{0}-1\right)\right) S+n_{0}\left(n_{0}-1\right) S\right] d_{\min }} \Rightarrow \\
P o S \leq \frac{n_{0}-1}{q+\frac{q-\left(n_{0}-1\right)}{n_{0}}}+\frac{q-\left(n_{0}-1\right)}{q+\frac{q-\left(n_{0}-1\right)}{n_{0}}} \cdot \frac{d_{\max }}{d_{\min }}
\end{gathered}
$$

Now notice that, since every node of the $n_{0}$ accesses replicas stored in all other $n_{0}-1$ nodes, it must be $n_{0}-1 \leq q$. Thus the first term can be at most 1 , while for $q=O(1)$, the second term is maximized to $\frac{d_{\max }}{2 d_{\min }}$ for $n_{0}=1$.

Simulation on Random Instances. We performed simulations on random instances of the general DSR game, that provide evidence of existence of efficient equilibria on average. Fig. 1 depicts the evolution of emipirically extracted values for PoS, PoA, and an average value of coordination ratio, as $d_{\max } / d_{\min }$ increases. We used an LWY(10) network of 64 nodes, with size $_{i}=5$, and $\left|R_{i}\right|=10$. For each node $i$ the request set $R_{i}$ was constructed by uniform random sampling of 10 objects out of a universe $U$ of 400 objects. Demand weights were taken 

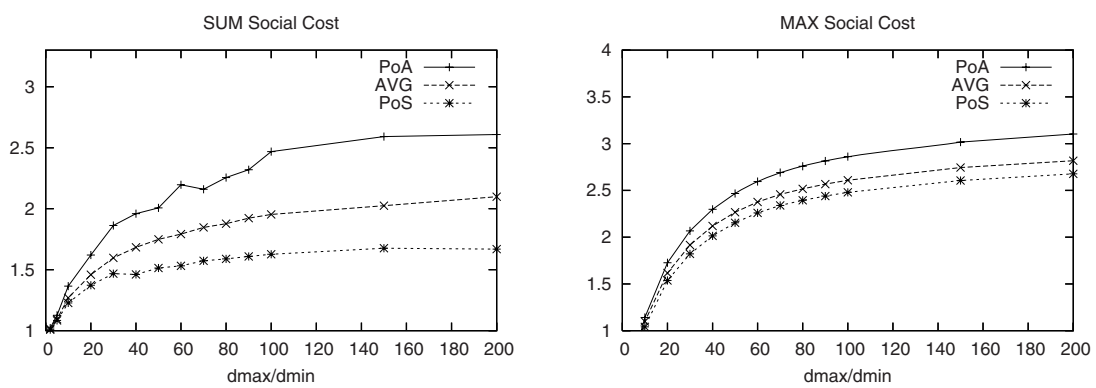

Fig. 1. Evolution of PoS, PoA, and average coordination ratio (AVG) with $d_{\max } / d_{\min }$

randomly in $(0,1]$ for each node. We took $d_{\min }=1$ and distributed 10 servers, each hosting 40 objects, uniformly within the range $\left[d_{\min }, d_{\max }\right]$ for each different value of $d_{\max }$.

Social optimum for SUM and MAX was obtained by solution of the Object Placement Problem ILP formulation described in [5], using GLPK 16. Each point of the diagrams was extracted using the maximum (for PoA), minimum (for PoS) and average (for AVG) social cost obtained over 1000 executions of algorithm DSR-EQ with randomly chosen node and object orderings per execution.

Two main observations can be extracted by these experiments. First, that the rate of growth of all coordination ratios degrades significantly as $d_{\max } / d_{\min }$ increases, thus giving the impression of a stabilization of the efficiency of the system. Second, that the range of different stable social cost values is quite narrow. These observations motivate a probabilistic analysis of the efficiency of equilibria, that constitutes an aspect of future work.

\section{Conclusions and Open Questions}

We studied the effects of selfish behavior in a distributed replication group with restricted storage capacity per node. We proved existence of pure strategy Nash equilibria for the related strategic game for networks with multiple origin servers, thus generalizing the result of [12. Although worst-case maximum and minimum social cost of equilibria were shown to be $\frac{d_{\max }}{d_{\min }}$ times the optimum social cost in the worst case, for $0 / 1$ demand weights and certain topologies a better upper bound of $\frac{d_{\max }}{2 d_{\min }}$ was shown for the Price of Stability. Simulations on random instances have shown that even the Price of Anarchy may grow much less rapidly than the ratio $d_{\max } / d_{\min }$.

We were not able to find a single example without any pure equilibria when arbitrary-sized objects are involved, or disprove existence of pure equilibria for more general topologies. We consider investigation of these situations as an aspect of future work, along with enrichment of the game model with other realistic features, such as bounded node degrees. 


\section{References}

1. Wessels, D., Claffy, K.: ICP and the Squid web cache. IEEE Journal on Selected Areas in Communications 16(3) (1998)

2. Fan, L., Cao, P., Almeida, J., Broder, A.Z.: Summary cache: a scalable wide-area web cache sharing protocol. IEEE/ACM Transactions on Networking 8(3), 281-293 (2000)

3. Stoica, I., Morris, R., Liben-Nowell, D., Karger, D.R., Kaashoek, M.F., Dabek, F., Balakrishnan, H.: Chord: A scalable peer-to-peer lookup protocol for internet applications. IEEE/ACM Transactions on Networking 11(1), 17-32 (2003)

4. Leff, A., Wolf, J., Yu, P.S.: Replication Algorithms in a Remote Caching Architecture. IEEE Transactions on Parallel and Distributed Systems 4(11), 1185-1204 (1993)

5. Baev, I., Rajaraman, R.: Approximation algorithms for data placement in arbitrary networks. In: Proceedings of the ACM-SIAM Annual Symposium on Discrete Algorithms (SODA), pp. 661-670 (2001)

6. Nash, J.F.: Non-Cooperative ganes. Annals of Mathematics 54, 286-295 (1951)

7. Osborne, M.J., Rubinstein, A.: A course in game theory. MIT Press, Cambridge (1994)

8. Chun, B., Chaudhuri, K., Wee, H., Barreno, M., Papadimitriou, C.H., Kubiatowicz, J.: Selfish caching in distributed systems: a game-theoretic analysis. In: Proceedings of the ACM Symposium on Principles of Distributed Computing (PODC) (2004)

9. Koutsoupias, E., Papadimitriou, C.H.: Worst-case Equilibria. In: Proceedings of the Symposium on Theoretical Aspects of Computer Science (STACS), pp. 404413 (1999)

10. Anshelevich, E., Dasgupta, A., Tardos, E., Wexler, T.: Near-optimal network design with selfish agents. In: Proceedings of the ACM Annual Symposium on Theory of Computing (STOC), pp. 511-520 (2003)

11. Anshelevich, E., Dasgupta, A., Kleinberg, J.M., Tardos, E., Wexler, T., Roughgarden, T.: The Price of Stability for Network Design with Fair Cost Allocation. In: Proceedings of IEEE Annual Symposium on Foundations of Computer Science (FOCS), pp. 295-304 (2004)

12. Laoutaris, N., Telelis, O.A., Zissimopoulos, V., Stavrakakis, I.: Distributed Selfish Replication. IEEE Transactions on Parallel and Distributed Systems 17(12), 14011413 (2006)

13. Fabrikant, A., Luthra, A., Maneva, E.N., Papadimitriou, C.H., Shenker, S.: On a network creation game. In: Proceedings of the ACM Symposium on Principle of Distributed Computing (PODC), pp. 347-351 (2003)

14. Chen, H., Roughgarden, T., Valiant, G.: Designing networks with good equilibria. In: Proceedings of the ACM-SIAM Symposium on Discrete Algorithms (SODA) (to appear, 2008)

15. Nisan, N., Roughgarden, T., Tardos, E., Vazirani, V.V. (eds.): Algorithmic Game Theory. Cambridge University Press, Cambridge (2007)

16. GNU Linear Programming Kit, http://www.gnu.org/software/glpk/ 